The University of Arizona

Department of Mathematics

\title{
Analysis of Equity Markets: A Graph Theory Approach
}

\author{
Joshua Rubin Abrams, José Celaya-Alcalá, Drew Baldwin \\ Ryan Gonda, Zhaoren Chen
}

May 16, 2017 


\section{Contents}

\begin{tabular}{lll}
\hline 1 & Introduction & 193
\end{tabular}

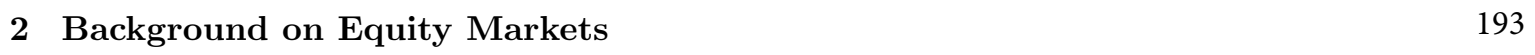

2.1 Stocks $\ldots \ldots \ldots \ldots \ldots \ldots$

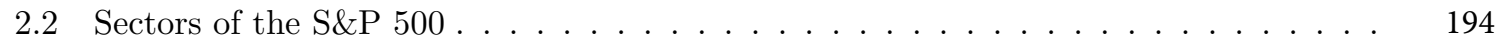

2.3 Terminology $\ldots \ldots \ldots \ldots \ldots \ldots$

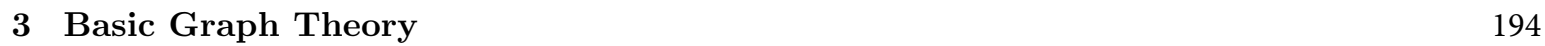

\begin{tabular}{lll}
\hline 4 & Assumptions & 196
\end{tabular}

$\begin{array}{|ll|}5 \text { Methods from the Paper } & 197\end{array}$

\begin{tabular}{lll}
\hline Analysis & 197
\end{tabular}

6.1 Data Collection . . . . . . . . . . . . . . . . . . . . . . . . . . 198

6.2 Spearman Rank Correlation . . . . . . . . . . . . . . . . . . . . . . . . . 198

6.3 Graph Statistics . . . . . . . . . . . . . . . . . . . . . . . . . . . . 199

$6.3 .1 \quad$ Degree Distribution $\ldots \ldots \ldots \ldots \ldots$

$6.3 .2 \quad$ High Degree Stocks . . . . . . . . . . . . . . . . . . . . . . . . . . . 199

$6.4 \quad k$-Core Analysis . . . . . . . . . . . . . . . . . . . . . . . . . . . . 200

6.5 Sector Graph . . . . . . . . . . . . . . . . . . . . . . . . . . . . . . 201

\begin{tabular}{lll}
\hline 7 & Future Development & 202
\end{tabular}

7.1 Directed Graph $\ldots \ldots \ldots \ldots \ldots \ldots$. . . . . . . . . . . . . . . . . 202

7.2 Short-Term Trading Tools $\ldots \ldots \ldots$. . . . . . . . . . . . . . . . . . . . . 202

7.3 Real Business Cycle Modeling . . . . . . . . . . . . . . . . . . . . . . . . . . 202

\begin{tabular}{lll}
\hline 8 & Conclusion & 203
\end{tabular}

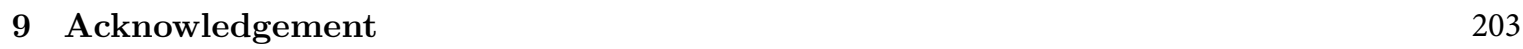


\begin{tabular}{ll}
\hline 0 Appendix A: Code & 205
\end{tabular}

11 Appendix B: Graphs 208

12 Appendix C: Tables 209 


\begin{abstract}
In this paper, we develop a characterization of the structure of the US Stock Market by studying how correlations between the various stocks and sectors of the market fluctuate. Through this characterization, we hope to identify the "strongest" of stocks, and sectors and thus identify which investments are safest. This analysis will allow us to provide an alternate investment strategy for those wishing to avoid long term risk in equity markets. This is done using a correlation based graph representing the stock market. The central finding of this study is that transportation sector, a subset of the industrial sector, is the best indicator of the market's fluctuation.
\end{abstract}

\title{
1 Introduction
}

The stock market may be one of the best examples of randomness in the world. Millions of shares are sold and bought each day by individuals worldwide. The potential for application of mathematical models and methods to understand market behavior is significant. Accounting for the current economic and political scenarios at the time of investment is an incredibly difficult task. This may be used to explain the current uneasiness of investing capital in equities markets. We explore the most efficient ways to maximize the possible return on equities while at the same time reducing exposure to the inherit volatility of the market. The work by Shirokikh, Pastukhov et al., which uses the techniques of graph theory and employs autoregressive models to accomplish this goal is the starting point of this study [1].

\section{Background on Equity Markets}

\section{$2.1 \quad$ Stocks}

A stock is like a partitioning of a company. A consumer can purchase one of these partitions, called a share. So if one purchases a share of, say, GOOGLE, you are part owner of the company. Hence use of the word "share". Colloquially, the words "stock" and "share" are used interchangeably. We will do the same for the remainder of this paper.

Stocks are traded like any other good; if more people buy stocks than sell them, prices increase. Alternatively, if more people sell than buy, the price decreases. Prices can be seen as information. As such, price movement can be interpreted as information moving between investors on the state of the market. One could draw analogies to a disease spreading through a population, or electricity through a network.

Stocks are listed and traded on exchanges. These exchanges include the NASDAQ, the New York Stock Exchange, and the London Stock Exchange, though the list is extensive. Overall market performance can be gauged by looking at indices [4. Some examples of indices are the S\&P 500, DJIA, and the DAX. Indices are weighted averages of the prices of various stocks in a subset of the market. So, if say the DJIA value is decreasing, then the value of multiple stocks are decreasing, which is indicative of poor market behavior.

In this paper, we focus on the $\mathrm{S} \& \mathrm{P} 500$ index which represents five hundred American companies with the biggest market capitalization. These companies may be thought of as the 'biggest', or most 
influential companies which would ideally reflect the state of the United States stock market.

\subsection{Sectors of the S\&P 500}

A sector is a subset of the overall market. There are 10 sectors in the S\&P 500 with each sector categorizing a type of industry in the US economy. Some examples included in the S\&P 500 are Health care, Technology, and Financial sectors. These sectors effectively break up the market into different sections, each of which deals with a factor of production in an economy. The idea of sectors allows us to globally analyze which parts of the market are strongest/most connected. For example, we can find sector trends in these ten sectors and measure the strength of each trend. This is crucial in our model as it will allow for more robust short term analysis. This will also allow us to analyze the behavior of markets in downturn and upturn trends, and help explain why financial crashes bring on recessions.

\subsection{Terminology}

Here we will define some terms we will use throughout the paper. The time series of a stock is one of its characteristics plotted against time, such as price against time. More technically, a time series $T_{s}(t)$ is a function $\mathbb{R} \rightarrow \mathbb{R}$ that gives the value of stock $s$ at time $t$. A stock's log returns are simply the logarithm of its returns. An ETF is an Exchange Traded Fund, it is a fund that is made up of investments in various commodities, stocks, or bonds. An ETF can be traded much like a stock.

\section{Basic Graph Theory}

This section is designed to be a lexicon for the reader. We discuss concepts of graph theory and how they apply to this paper's approach to this project.

We consider a simple graph $G=(V, E)$ to be a tuple, where $V$ is a set of vertices (or nodes) and $E$, a set of edges, is a subset of $V \times V$. A graph is comprised of edges that connect vertices. The notation $(u, v) \in E$, means that node $u$ shares an edge with node $v$. We say that two nodes are connected if they share an edge.

The first part of our research focuses on undirected graphs. A graph is undirected if whenever $(u, v) \in E,(v, u) \in E$ too. We denote a mutual connection between two nodes by a straight line that connects those two nodes. Thus, one way connections are not possible by this definition. Later in section 7.1, we address the case of directed graphs. These are graphs whose edges point in only one direction, and so allow one way connections. In the context of this paper, vertices in a graph are stocks that are connected by an edge. Directed graphs will be used to explore how one stock may influence another; we represent this relationship by having the influential stock point to the influenced stock.

We can describe connections of a graph using an adjacency matrix. The adjacency matrix is an $n \times n$ matrix, where $n$ is the number of nodes in a graph. If one were to enumerate the nodes of the graph, the connections of the graph are described by placing a 1 in entries whose coordinates correspond to the nodes that have a connection. Otherwise, two nodes that are not connected get a 0 in that entry. Often, a node is not connected to itself, as in the following example, but this is not 
a requirement of the definition.

A weighted graph is a graph whose edges are assigned values that represent the importance (or weight) of that edge. The adjacency matrix that describes the weighted edges is called a weighted adjacency matrix. See Figure 1 for an example of an undirected weighted graph and Figure 2 for the corresponding weighted adjacency matrix that describes the connections of Figure 1.

One should note that the adjacency matrix produced for the analysis of stocks in this paper is a weighted adjacency matrix whose entries reflect the correlation between any pair of these stocks. Thus we expect a value of 1 all along the diagonal since every stock is directly correlated with itself.

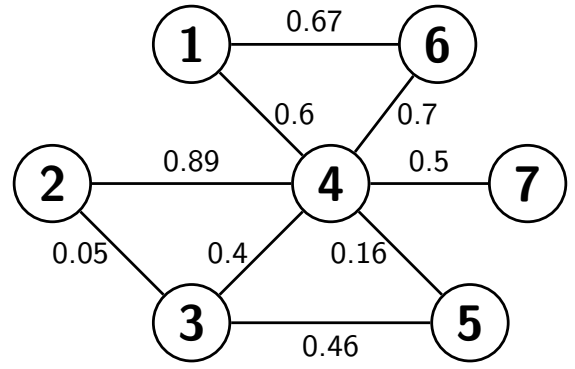

Figure 1: Weighted Undirected Graph

$$
\left[\begin{array}{ccccccc}
0 & 0 & 0 & 0.6 & 0 & 0.67 & 0 \\
0 & 0 & 0.05 & 0.89 & 0 & 0 & 0 \\
0 & 0.05 & 0 & 0.4 & 0 & 0 & 0 \\
0.6 & 0.89 & 0.4 & 0 & 0.16 & 0.7 & 0.5 \\
0 & 0 & 0.46 & 0.16 & 0 & 0 & 0 \\
0.67 & 0 & 0 & 0.7 & 0 & 0 & 0 \\
0 & 0 & 0 & 0.5 & 0 & 0 & 0
\end{array}\right]
$$

Figure 2: Weighted Adjacency Matrix corresponding to Figure 1

We refer to the degree of a node to be the number of connections a node has.

A degree matrix is a $n \times n$ matrix, whose diagonal entries correspond to the degree of the $i^{t h}$ node and whose off-diagonal entries are all zero. The combination of these two matrices (degree and adjancency matrix) describe all qualitative aspects of the graph.

Graphs can be broken up into subgraphs. Each subgraph is a subset of the original graph. We say that node $u$ is path-connected to node $v$ if there exists $u_{1}, u_{2}, \ldots, u_{k} \in V$ such that $u$ is connected to $u_{1}, u_{i}$ is connected to $u_{i+1}$ for all $i \in\{1, \ldots, k-1\}$, and $u_{k}$ is connected to $v$. A connected component of an undirected graph is a subgraph in which any two nodes are path-connected and there exists no edge between any node in the subgraph to a node outside the subgraph.
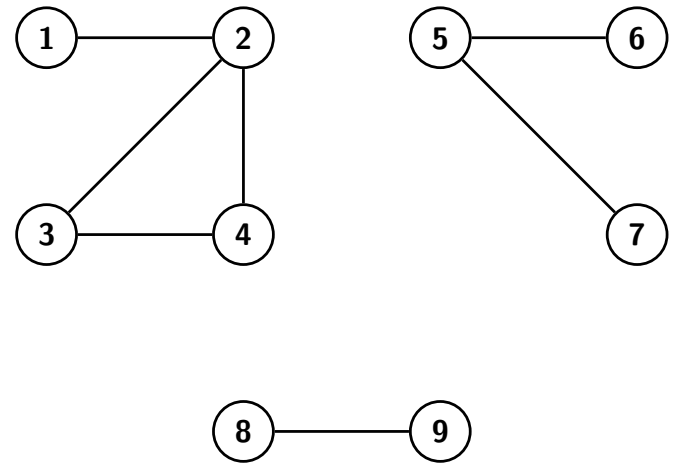

Figure 3: Undirected graph with 3 connected components

$$
\left[\begin{array}{lllllllll}
0 & 1 & 0 & 0 & 0 & 0 & 0 & 0 & 0 \\
1 & 0 & 1 & 1 & 0 & 0 & 0 & 0 & 0 \\
0 & 1 & 0 & 1 & 0 & 0 & 0 & 0 & 0 \\
0 & 1 & 1 & 0 & 0 & 0 & 0 & 0 & 0 \\
0 & 0 & 0 & 0 & 0 & 1 & 1 & 0 & 0 \\
0 & 0 & 0 & 0 & 1 & 0 & 0 & 0 & 0 \\
0 & 0 & 0 & 0 & 1 & 0 & 0 & 0 & 0 \\
0 & 0 & 0 & 0 & 0 & 0 & 0 & 0 & 1 \\
0 & 0 & 0 & 0 & 0 & 0 & 0 & 1 & 0
\end{array}\right]
$$

Figure 4: Unweighted Adjacency Matrix corresponding to Figure 3 
The graph Laplacian, denoted $L$, is defined in the following way:

$$
L:=D-A
$$

where $D$ refers to the degree matrix and $A$ refers to the adjacency matrix of a given graph. We can describe many qualitative characteristics of a graph using a graph Laplacian. The following is a list of properties of the graph Laplacian.

- If the graph $G$ is undirected, then its adjacency matrix and graph Laplacian are symmetric.

- The sum of the rows of the graph Laplacian is always 0 , because the degree of each node matches the number of nodes it is adjacent to.

- It follows that zero is always an eigenvalue to the eigenvector of all ones in every entry.

- The eigenvalues of the graph Laplacian are nonnegative real numbers.

- The algebraic multiplicity of the 0 -eigenvalue describes the number of connected components of a graph.

- The smallest positive eigenvalue gives us a notion of how "connected" the graph is. It is related to what is commonly known as Cheeger's constant [2].

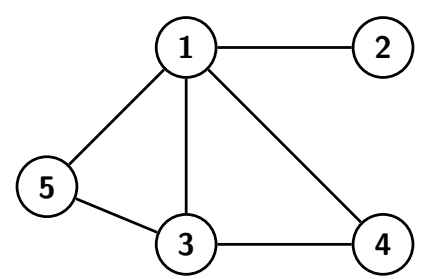

Figure 5: Undirected graph

$\left[\begin{array}{lllll}0 & 1 & 1 & 1 & 1 \\ 1 & 0 & 0 & 0 & 0 \\ 1 & 0 & 0 & 1 & 1 \\ 1 & 0 & 1 & 0 & 0 \\ 1 & 0 & 1 & 0 & 0\end{array}\right]$

Figure 6: Unweighted Adjacency Matrix corresponding to Figure 5

$$
\left[\begin{array}{ccccc}
4 & -1 & -1 & -1 & -1 \\
-1 & -1 & 0 & 0 & 0 \\
-1 & 0 & 3 & -1 & -1 \\
-1 & 0 & -1 & 2 & 0 \\
-1 & 0 & -1 & 0 & 2
\end{array}\right]=\left[\begin{array}{ccccc}
4 & 0 & 0 & 0 & 0 \\
0 & 1 & 0 & 0 & 0 \\
0 & 0 & 3 & 0 & 0 \\
0 & 0 & 0 & 2 & 0 \\
0 & 0 & 0 & 0 & 2
\end{array}\right]-\left[\begin{array}{ccccc}
0 & 1 & 1 & 1 & 1 \\
1 & 0 & 0 & 0 & 0 \\
1 & 0 & 0 & 1 & 1 \\
1 & 0 & 1 & 0 & 0 \\
1 & 0 & 1 & 0 & 0
\end{array}\right]
$$

Figure 7: Graph Laplacian corresponding to graph in Figure 5

\section{Assumptions}

Before delving into the problem and existing methods to analyze market strength, we point out the following critical assumptions for the purposes of this paper:

- The financial market is efficient. In other words, all market information is available to every participant at any given time, and stocks are perfectly priced. This assumption provides us the capability to use the price alone to analyze each stock. 
- Stock prices move continuously, allowing us to represent the stock market as a dynamical system, and employ time series shifting.

- There is a correlation between the movement of prices of every stock as well as the performance of each sector. It measures statistical relationships between two or more random variables or observed data values.

- Each sector has at least one major group of stocks, which strongly correlate with each other, and the groups are the major drivers in each sector. Later we define them as cluster groups.

- A stock may influence another stock within a ten day period. This is used for the implementation of our time series shifting in the directed graph.

\section{Methods from the Paper}

In [1], Shirokikh, Pastukhov et al. make use of graph theory and statistics to compute the relationship between stocks. The Spearman Rank correlation coefficient, denoted $\rho$, is defined in the following way:

$$
\rho=\frac{\sum_{i}\left(x_{i}-\bar{x}\right)\left(y_{i}-\bar{y}\right)}{\sqrt{\sum_{i}\left(x_{i}-\bar{x}\right)^{2} \sum_{i}\left(y_{i}-\bar{y}\right)^{2}}}
$$

where $x=\left(x_{1}, \ldots, x_{n}\right)$, and $y=\left(y_{1}, \ldots, y_{n}\right)$ are the time series or log returns of any two stocks $x$ and $y . \bar{x}$ and $\bar{y}$ are the average values of the entries of $x$ and $y$. The above correlation acts as a rank-based approach of correlating stocks depending on price. The Spearman rank correlation is analogous to an inner product between two vectors. Correlations take on any value in the interval $[-1,1]$, where a correlation of 1 is a direct correlation, a correlation of -1 is an inverse correlation, and a correlation of 0 represents no correlation. The authors of [1] recorded the correlations between every pair of stocks in a weighted adjacency matrix.

The Spearman rank correlation was studied and compared to the Pearson rank correlation, which is simply another method to quantify correlation between to stocks [3]. We chose to use the Spearman correlation, because it can represent correlations independent of outliers and nonlinearities, where the Pearson correlation cannot.

While we should note that the authors of [1] used an undirected graph, they were still able to obtain a set of stocks with the highest correlations. However, these have no relation to sectors. We remedy this problem by proposing a novel approach to modeling correlation between equities. While this field uses varying degrees of statistics, [1] took a more visual approach with graph theory. This methodology provides a more global view of how the different sectors of the market are interacting, improving the accuracy of short term analysis.

\section{Analysis}

As mentioned above, the paper [1] uses statistics and graph theory extensively. Here, we go into detail about how we used these ideas and/or modified them for our purposes. Also, we will present how we wish to expand on the paper, and explain how this analysis will culminate in an investment strategy that minimizes long term risk in equity markets. 


\subsection{Data Collection}

We collected our data from the Wharton Research Data Services (WRDS), an online database of financial information 5. We did this for every stock that appeared in the S\&P 500 from January 1, 2008 to December 31, 2015. Due to changes in the composition of the S\&P 500 during this time frame, the data set included a total of 922 unique stocks with a large variance in the number of recorded days. We accounted for this variance by setting the Spearman rank correlation coefficient to 0 if the intersection length of two stocks' date lists was less than one sixth of the maximum possible length for the given period. This is to ensure that correlations are not predicted where there is not enough information.

\subsection{Spearman Rank Correlation}

We constructed each stocks time series using the value of the stock's daily returns, without dividends; we call this value the RETX value of the stock. We exclude dividends to allow us to focus purely on stock price due to trading between investors, and not from executive decisions on the part of the company.

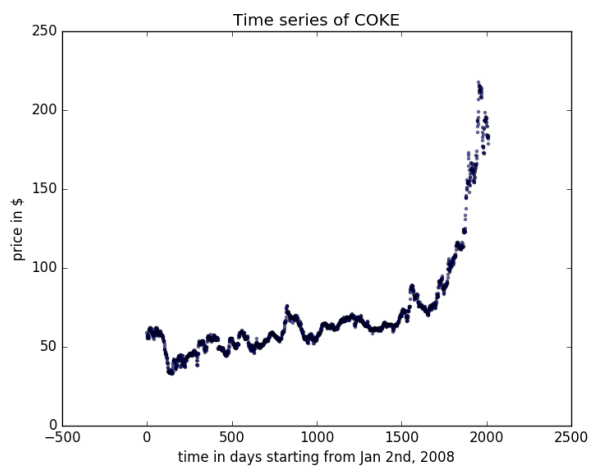

Figure 8: Example time series for Coca Cola

We then partitioned the data into 5 time periods: 2008-2009, 2010-2011, 2012-2013, 2014-2015 and 2008-2015.

As mentioned above, we found the intersection of the two stocks data list. If the length of this list was less than one sixth of the maximum possible length for the given period, a 0 was placed in the respective cells of the adjacency matrix. We deemed this to be a reasonable length of time for which stocks could truly be correlated. Stocks that intersect in less time may only happen to be correlated. Otherwise, each time series was ranked and the Spearman rank correlation coefficient $(\rho)$ was calculated and placed in the respective cells of the adjacency matrix.

Finally, we applied various minimum correlation thresholds defined by $\theta$. To apply the threshold, we transform the adjacency matrix by placing a 1, which represents an edge, in cells where $\rho>\theta$ and a 0 otherwise. We looked at $\theta=0.1,0.2, \ldots 0.9$. $\theta$ is a measure of the stringency of stocks in the adjacency matrix. A $\theta$ value which is too low will result in noisy data, inhibiting meaningful results. Similarly, a $\theta$ value which is too high will not have the sufficient data to perform our analysis. We found $\theta=0.6$ to be an ideal threshold; we still had on the order of thousands of data points for each 
2-year period and kept up to $10 \%$ of the information for each 2-year period. Keeping information for a $\theta$ value too low (e.g. 50\%) includes pairs of stocks that are loosely correlated. Please refer to tables 20 and 21 in Appendix $\mathrm{C}$ to view distribution of data that remained after choosing $\theta$ values.

\subsection{Graph Statistics}

\subsubsection{Degree Distribution}

The degree distribution of the graph's nodes can be used to describe connectivity patterns of the overall graph. We found that our degree distribution closely followed a power - law, that is, $\mathbb{P}(k) \propto$ $k^{-\gamma}$ where $\mathbb{P}(k)$ is the frequency of nodes with degree $k$. This is the case in many real-world networks (Shirokikh et al. 2013). This served as a check for accuracy, if we were incorrect in our approach then this power law would not have been evident. A comparison of our results and the expected power law distribution is shown below

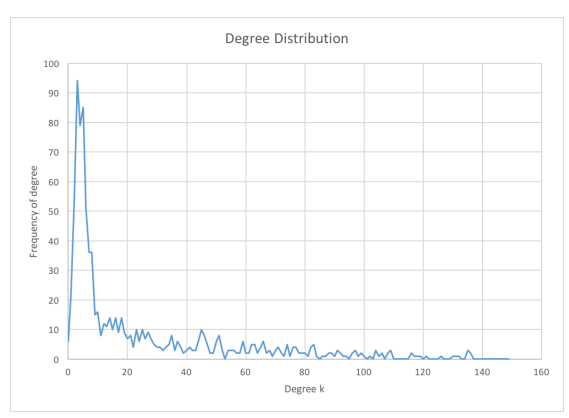

Figure 9: Generated degree distribution

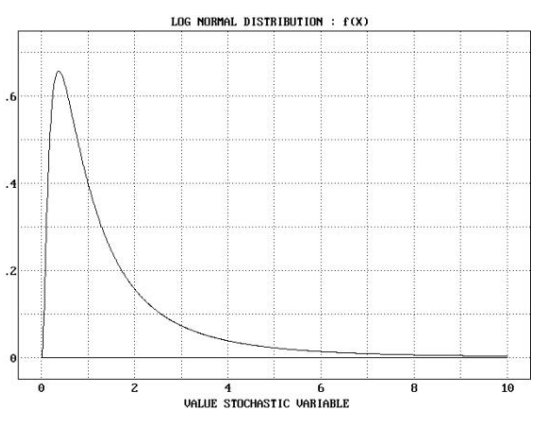

Figure 10: Power law distribution

\subsubsection{High Degree Stocks}

In order to determine which stocks have the largest impact on the overall market, we simply looked at the top 10 stocks with the highest degree in each period.

We found that, for a threshold $\theta=0.6$, in 2008-2009, 8 of the 9 highest degree stocks were from the Finance sector, while the other stock is no longer listed. For 2010-2011, we found there were new additions of stocks from the consumer defensive and industrial sectors. Then, for 2012-2013, the most correlated stocks were medium risk ETFs, with riskier ETFs falling in the 2014-2015 period.

Due to changes in the composition of the S\&P 500 for each period, the number of stocks that existed for a significant duration over a given period varied. Therefore, in the following chart, we calculated the degree of each stock as a percent of the maximum degree for that period. The maximum degree being the number of stocks that existed in that period. 


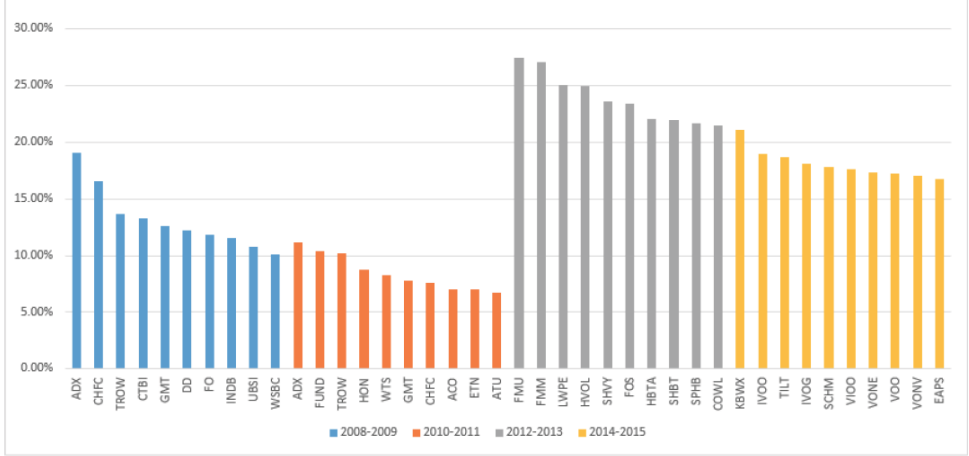

Figure 11: Degree of most correlated stocks as percent of maximum degree for period $(\theta=0.6)$

\section{$6.4 \quad k$-Core Analysis}

To potentially highlight additional structural properties of the graph, we move from the regular connected components, to the tight network clusters of $k$-cores. We chose $k$-cores as oppose to analyzing cliques, where all nodes are pairwise connected, due to computational efficiency. This is important since market analysis tends to deal with large data sets in time-critical settings.

A $k$-core is a subgraph containing all the remaining nodes after recursively removing nodes of degree less than $k$. To find the $k$-cores, an algorithm was used that recursively removed nodes with less than $k$ edges until all remaining nodes had $k$ edges. In the following example, starting with the graph in Figure 12, if we wanted to find the 3-core of the graph we would start by removing all nodes with degree less than 3 , which would be node 6 . We then repeat, and because of the previous removal, node 5 is now of degree 2, so we remove it. We are left with the graph in Figure 13.

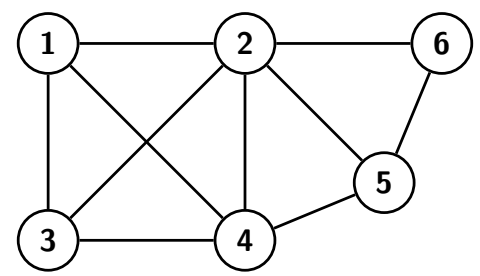

Figure 12: Undirected graph

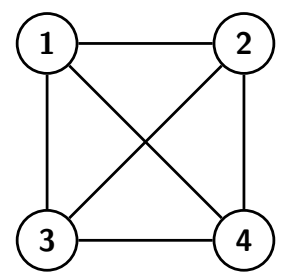

Figure 13: 3-core of graph in Figure 12

After computing the $k$-cores, we found the degeneracy of the graph. This is the maximum $k$ such that the graph contains a non-empty $k$-core. As noted for high degree stocks, a different number of stocks existed in each period and therefore we define the maximum degeneracy for a given period as the number of stocks that existed in that period. The plot below summarizes how, in each period, the degeneracy of the graph evolved as $\theta$ increased. 


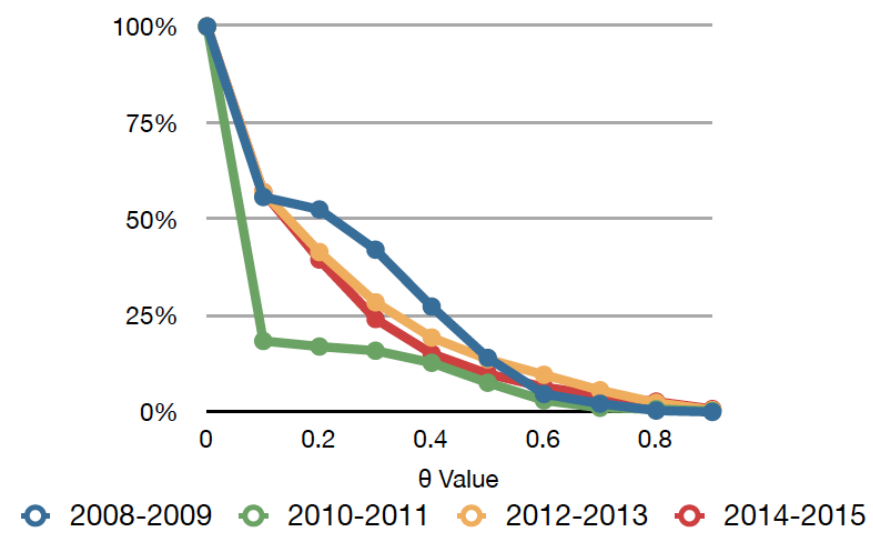

Figure 14: Degeneracy for each $\theta$ as percent of maximum degeneracy for period

This graph suggests that, during the recession period (2008-2009), a large cluster of loosely correlated stocks existed. Then, following the recession period (2010-2011), this cluster dramatically reduced in size, before stabilizing. One possible explanation for this behavior is that, during the recession period, many investors were selling shares to avoid losses. This could have resulted in a market wide drop. Then, for the next couple years, due to fear, activity in the market was markedly reduced.

\subsection{Sector Graph}

The sector graph allows us to globally visualize how each sector is interacting. It is paramount to the goal of our project to be able to quickly determine where the most connections are under the economic conditions at the time. By way of graph structure it will theoretically be easy to see when structural instabilities may be occurring in one sector and is thus likely to occur a downswing trend.In the period 2008-2009 the finance sector was the lowest correlated sector. We conclude this is due to the accelerating negative returns this sector was encountering in 2007-2008 from the recession in the US economy.

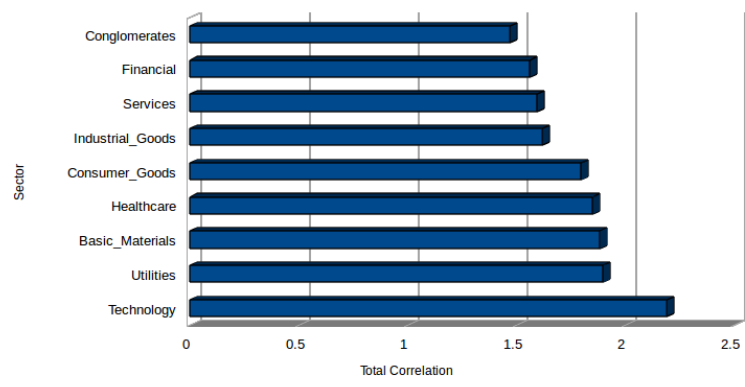

Figure 15: Total Sector correlation between 2008 and 2009

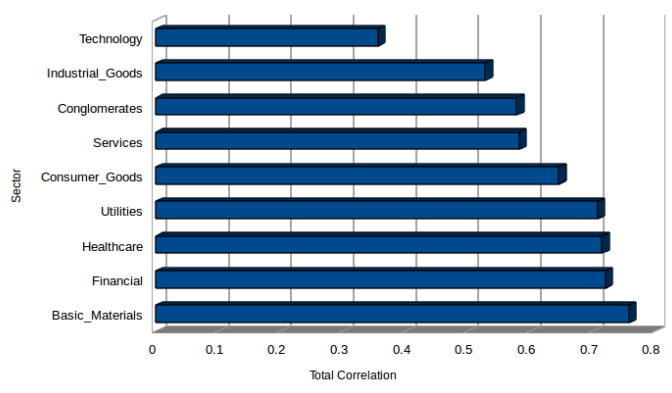

Figure 16: Total Sector correlation between 2010 and 2011 


\section{Future Development}

\subsection{Directed Graph}

Consider a time series $T_{s}(t): \mathbb{R} \rightarrow \mathbb{R}$ that gives the value of stock $s$ at time $t$. In practice, we won't have an exact function, but a data vector $\mathbf{T}_{s}$ for the stock, where the $k^{t h}$ entry is the value of the stock at time $k$. Define $\mathbf{T}_{s,[a, b]}$ to be the vector containing entries $a$ through $b$ of $\mathbf{T}_{s}$.

We have the Spearman-Rank function $S\left(\mathbf{T}_{1}, \mathbf{T}_{2}\right)$ which maps a pair of time series to a number between -1 and 1. Take two time series $\mathbf{T}_{s}$ and $\mathbf{T}_{r}$, of the same length $l$. Take $a=0$ and $b=l-N$, where $N$ is the maximum number of days before a stock can influence another. We make the assumption that $N=10$ suffices, but further research could determine what optimal $N$ is. We define the modified Spearman-Rank function:

$$
S_{M}\left(\mathbf{T}_{s}, \mathbf{T}_{r}\right)=\max \left\{S\left(\mathbf{T}_{s,[a, b]}, \mathbf{T}_{r,[a+k, b+k]}\right)\right\} \text { for } k \in\{0, \ldots, N\}
$$

We also define the max-delay function:

$$
M\left(\mathbf{T}_{s}, \mathbf{T}_{r}\right)=k \in\{0, \ldots, N\} \ni S\left(\mathbf{T}_{s,[a, b]}, \mathbf{T}_{r,[a+k, b+k]}\right) \geqslant S\left(\mathbf{T}_{s,[a, b]}, \mathbf{T}_{r,[a+i, b+i]}\right) \forall i \in\{0, \ldots, N\}
$$

The modified Spearman-Rank function allows us to define a directed graph, this is because unlike the Spearman-Rank function where $S\left(\mathbf{T}_{1}, \mathbf{T}_{2}\right)=S\left(\mathbf{T}_{2}, \mathbf{T}_{1}\right)$, the order of the arguments matter. If the modified Spearman-Correlation is high from $\mathbf{T}_{1}$ to $\mathbf{T}_{2}$, then $\mathbf{T}_{1}$ influences $\mathbf{T}_{2}$, while the reverse might not be true. In the paper by Shirokikh, Pastukhov et al., [1] mention the fact that their graph is undirected, and cannot show influence, so this method will hopefully fill this gap perfectly. In summary, this method will allow us to get a better picture of the market and the relationships between stocks, sectors etc.

\subsection{Short-Term Trading Tools}

Short term trading tools will play a pivotal role in the actual implementation of our model. Though our model can identify the sectors where the most robust analysis will be located it cannot be employed to make returns on individual stocks. Through short term trading tools such as Bollinger Bands, relative strength indicators, and ultimate oscillators etc. we will be able to (theoretically) better predict where a stock will be in a short time interval. The nature of short term trading tools is not one of high accuracy, usually due to weak market infrastructure and poor macroeconomic conditions, however our model should lesson the effects of external market forces and allow the analysis to rely purely on internal stock information. This will lead to a higher percentage of winning trades on the part of the investor.

\subsection{Real Business Cycle Modeling}

The term business cycle describes the booms and busts an economy encounters over a period of time. Macroeconomics devotes much of its research to this area, and we believe our project may lend itself to furthering knowledge in this area. We are fortunate enough to have data from the most recent financial crises in 2008, so we performed analysis to obtain meaningful results. The

data shows that previous to the 2008 financial crash, which occurred in October, the finance sector was the lowest correlated sector to the other 9 sectors. Interestingly enough, as the graph shows the 
finance sector composed more than 80 percent of the highest correlated stocks from 2008-2009. We recall that the spearman rank index is based upon RETX values, which indicated that the finance sector was composed of stocks that were generating returns which were highly similar. However, due to what we call inherent market weakness in the finance sector these values were all negative leading up to this period.Using this analysis we may generalize what conditions precede a recession and which precede a bull market.

\section{Conclusion}

The results that we obtained from our analysis matched what happened in the stock market through the time period we studied (2008-2015). We accomplished our goal of constructing a model for improving short term analysis. For the time period 2008-2009, our model showed that the transportation sector, a subset of industrial's, was the best candidate. In the following period of 2010-2011, the industrial sector was the highest returning sector. Furthermore, during 2008-2009, eight of the ten stocks with the greatest number of correlations were in the finance sector, with the other two stocks being DuPont which is listed in the industrial sector, and FO which is no longer listed on the exchange. During 2010-2011, many finance stocks remain as the highest degree stocks. However, there were more industrial and consumer defensive stocks who also had greater number of correlations. Thus, in the period of the stock market crash, most of the highly-correlated stocks were in the finance sector, leading to an isolation of finance stocks from all other stocks. In addition, the increasing negative returns of the finance stocks during 2008-2009 show that a financial crash occurred. Following the crash, we witnessed a flocking of ETF's (exchange traded funds), because investors wished to capture more of the volatility in the market recovery while hedging with safer stocks. As noted previously, the finance sector composed the most critical portion of the degree distribution, when the financial crises hit the financial sector, the result was a recession in the overall market.

Further research would include inducing a shock in the market by lowering all correlations of a sector with its neighbors due to increased negative returns to track the correlations, and returns of other sectors over time. One could investigate: which sectors have the greatest affect on the market, how long it would take for the market to stabilize, which stocks are most resistant to market crashes, and which stocks recover the most after the market crash i.e. identify the best stocks to invest in.

\section{Acknowledgement}

This project was mentored by Tova Brown, whose help is greatly appreciated. We thank Dr. Hermi for his guidance. Support from a UA TRIF (Technology Research Initiative Fund) grant to J. Lega is also acknowledged.

\section{References}

[1] Shirokikh, Oleg, Grigory Pastukhov, Vladimir Boginski, and Sergiy Butenko. Computational Study of the US Stock Market Evolution: A Rank Correlation-based Network Model. Computational Management Science Comput Manag Sci 10.2-3, (2013): 81-103. 
[2] Chung, F. R. K. Laplacians of graphs and Cheeger inequalities. math.ucsd.edu. N.p., n.d. Web. 3 May 2016. http://www.math.ucsd.edu/ fan/wp/cheeger.pdf.: Chapter 2

[3] Jan Hauke, Tomasz Kossowski. Comparison of Values of Pearson's and Spearman's Correlation Coefficients on the Same Sets of Data. Quaestiones Geographicae. June 2011. Volume 30, Issue 2, Pages 87-93, ISSN (Print) 0137-477X, DOI: 10.2478/v10117-011-0021-1.

[4] "Market Indices." sec.gov, https://www.sec.gov/fast-answers/answersindiceshtm.html. Accessed 16 May 2017.

[5] Wharton Research Database Services, Wharton School of Business, https://wrdsweb.wharton.upenn.edu/wrds/. Accessed 16 Feb 2016. 


\section{Appendix A: Code}

All Data Analysis for this project was done in Python. Some graphs and figures were generated using Excel. We created tools for the following:

- Plotting a time series for a stock

- Calculating the Spearman-Rank Correlation

- Creating the adjacency matrix for our graph

- Enforcing a Theta Threshold on an adjacency matrix

- Finding the k-cores in our network

- Determine the degeneracy of the graph

- Graphing an an adjacency matrix (2D and 3D)

- Determining correlations between sectors

- Calculating the Degree Distribution of our graph

- Calculating the Edge Density of our graph

- Various other minor scripts

Our code is available at: https://bitbucket.org/Team_Name_Goes_Here/stock-analysis

Code to generate the Spearman Adjacency Matrix for a given starting year and ending year.

\# for each name in stocknames

for a in xrange(2, len(stocknames)):

row $=[]$

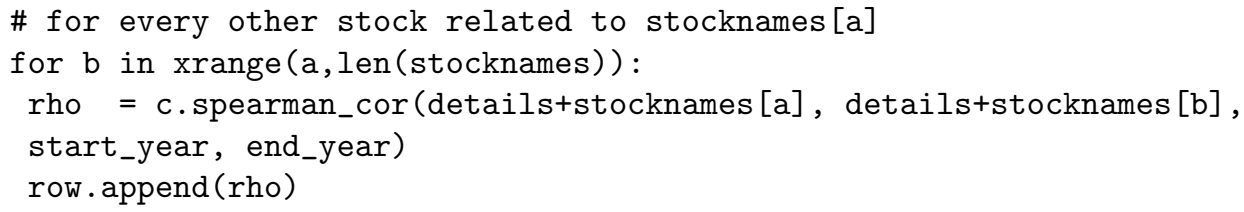

The code to find which stocks are in a given $k$-core is shown below:

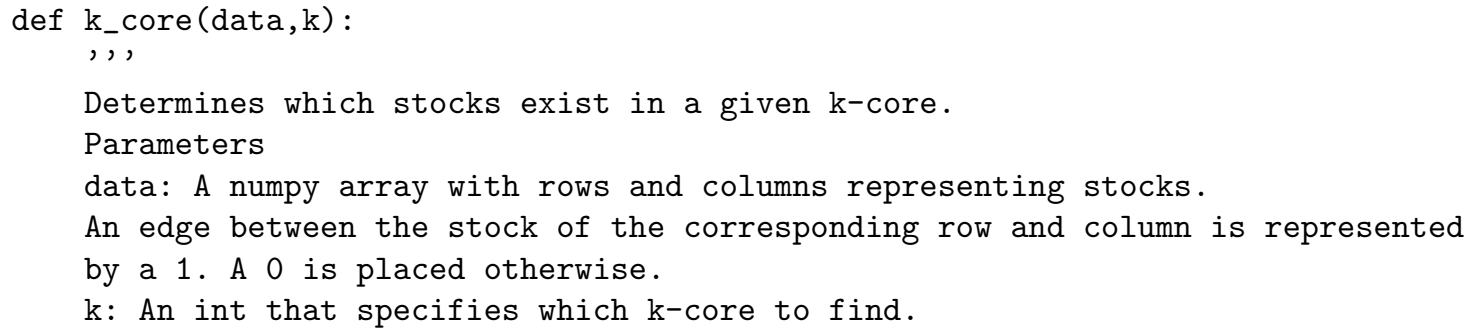




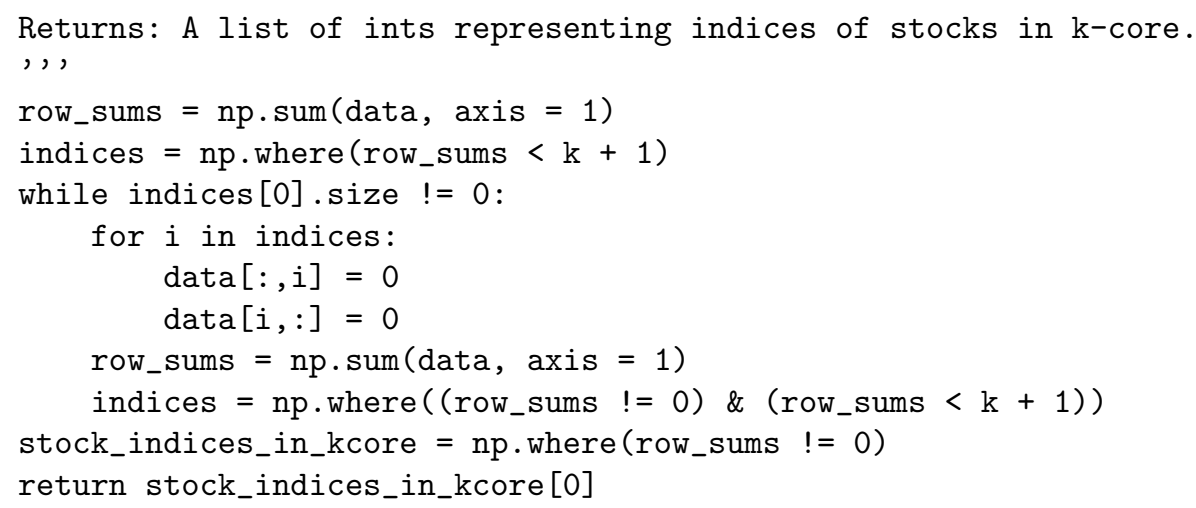

Snippet of code to calculate correlations between sectors

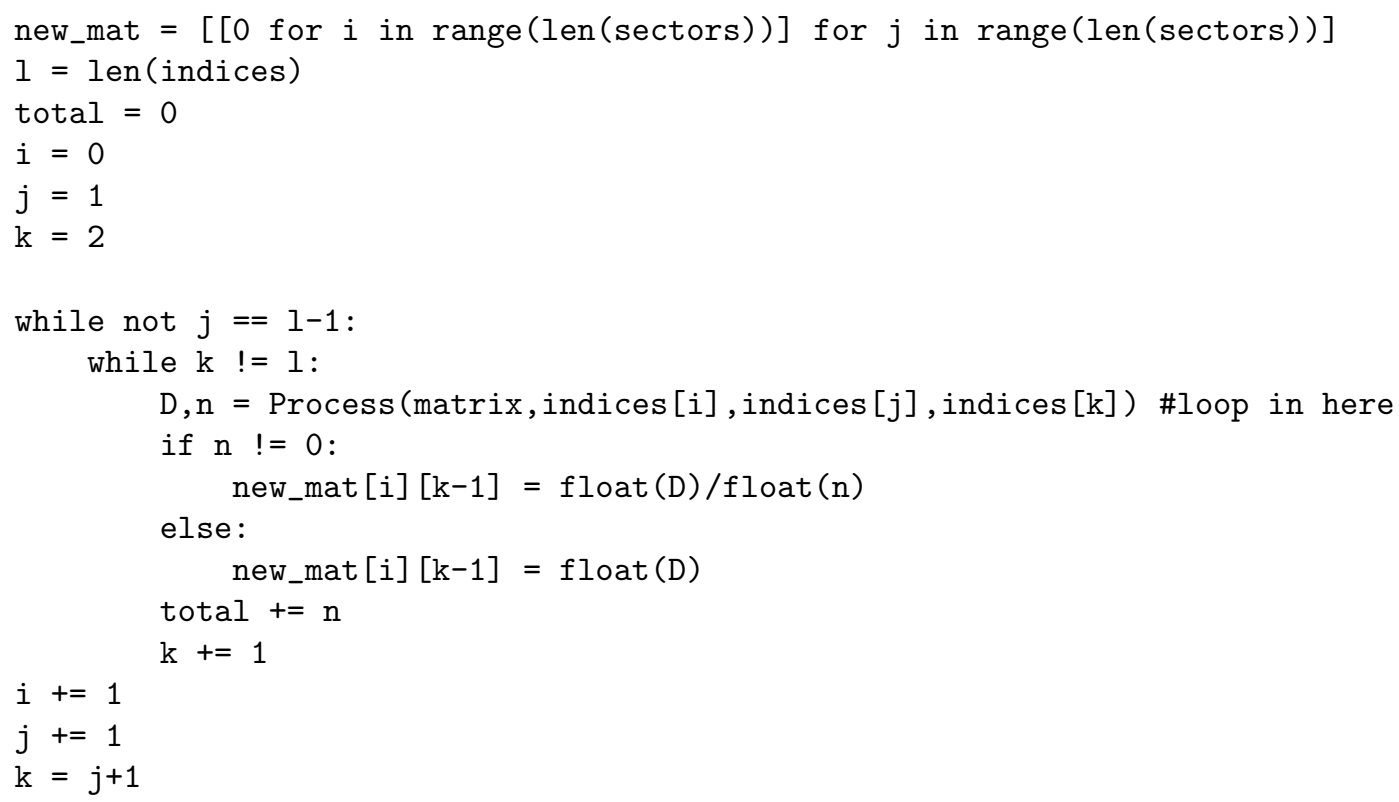

To generate the degree distribution

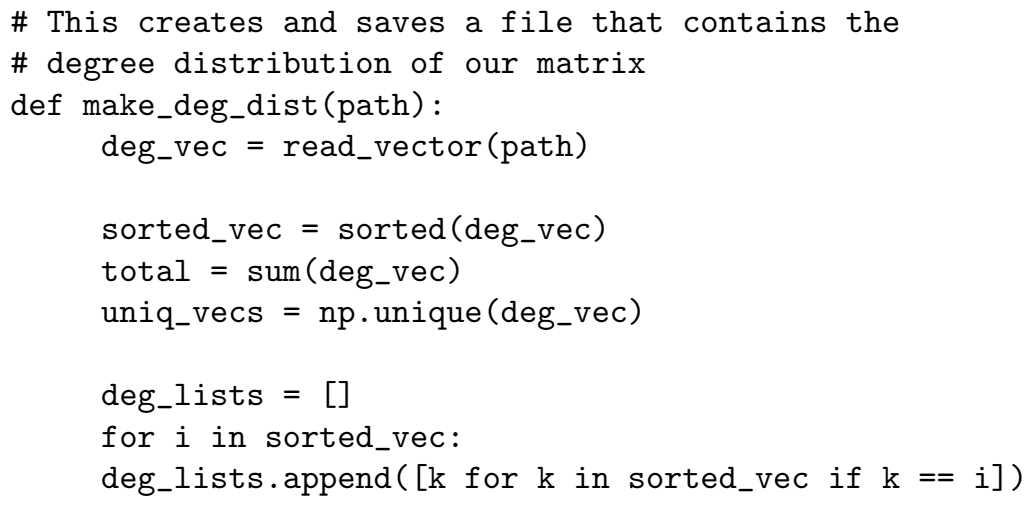




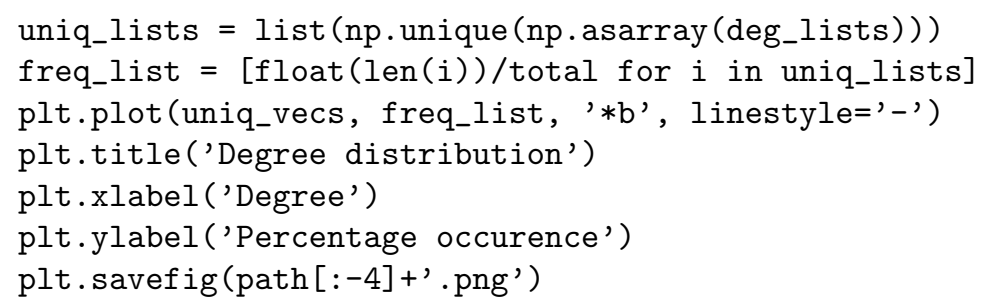

The following functions were used to calculate the eigenvalues of a given matrix and for the graph laplcian, identify the number of connected components and connectivity of the graph

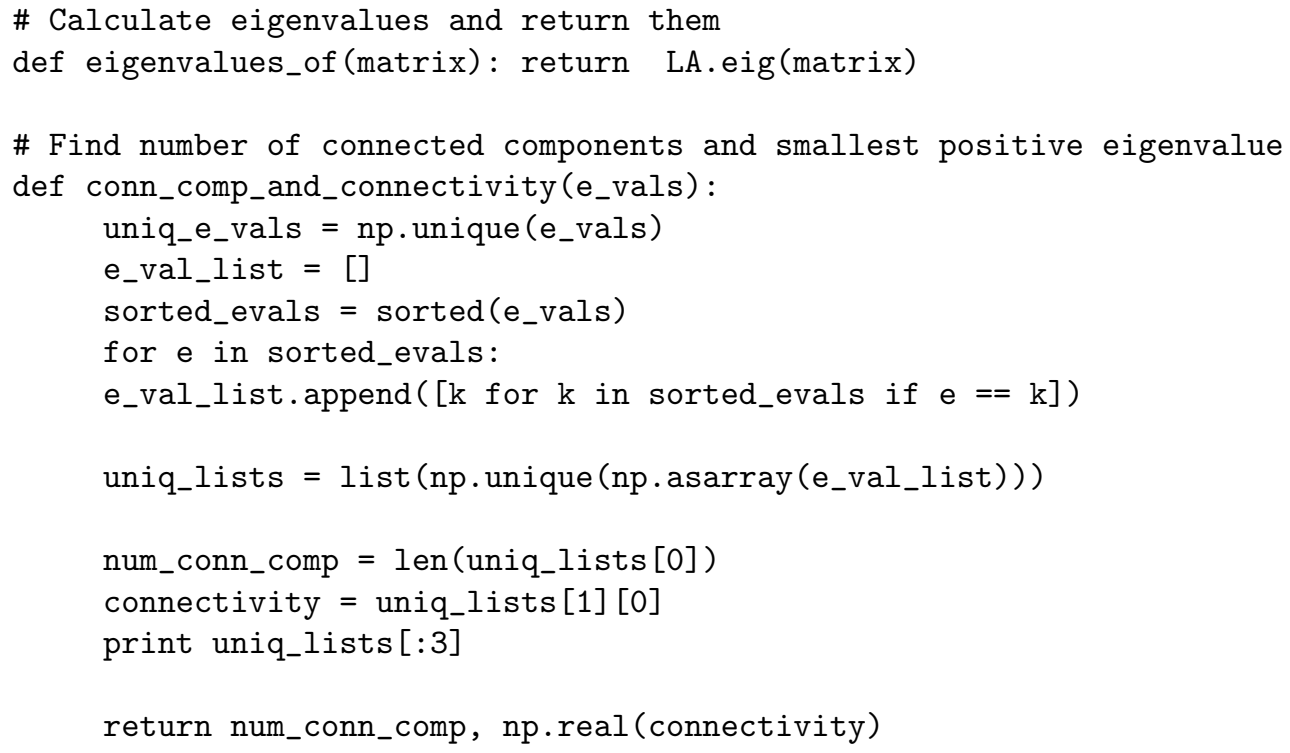

Part of code used to strip data about sectors from yahoo finance

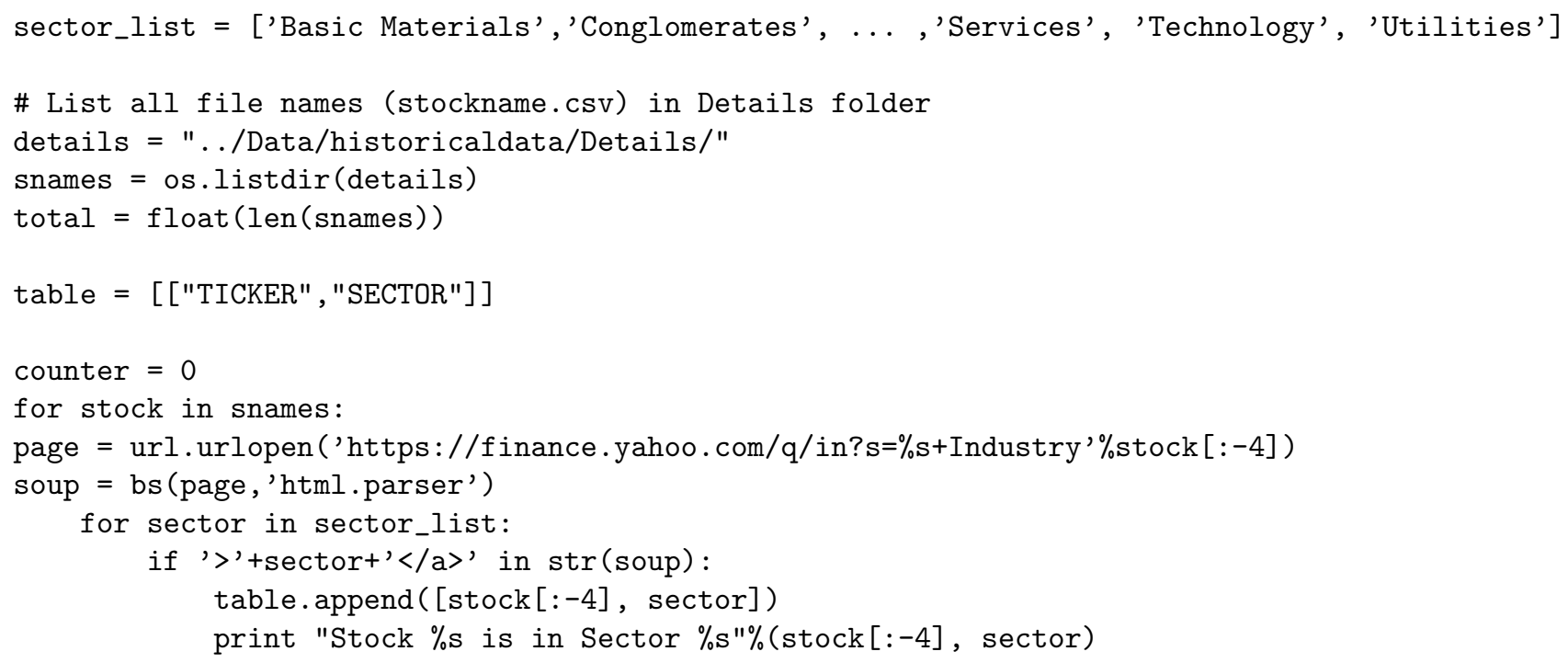


else:

table.append([stock [:-4], "NONE"])

write_content(table, "sector_data.csv")

\section{Appendix B: Graphs}

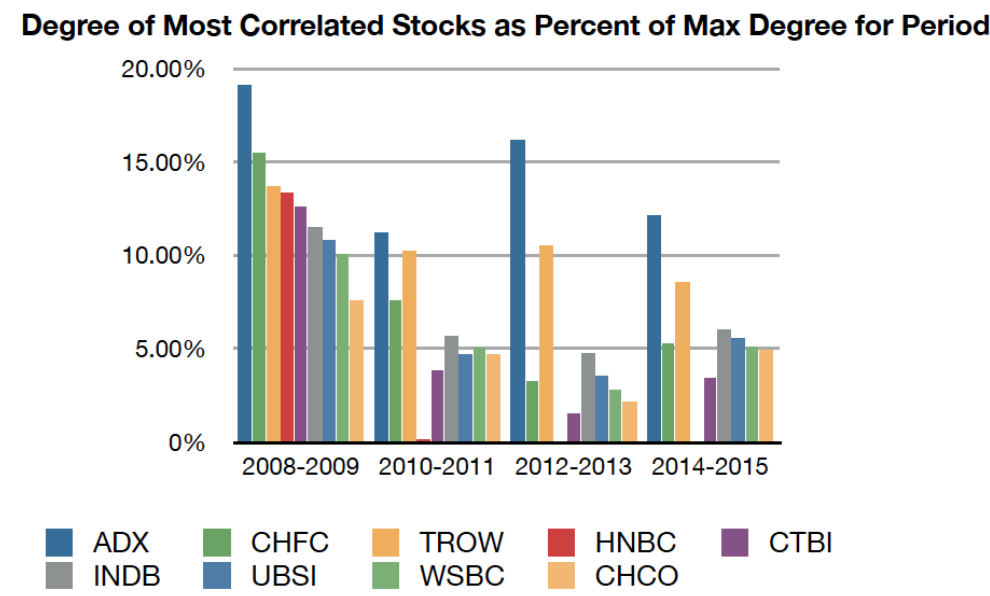

Figure 17: Top degree stocks in 2008-2015 are plotted for each time period. 


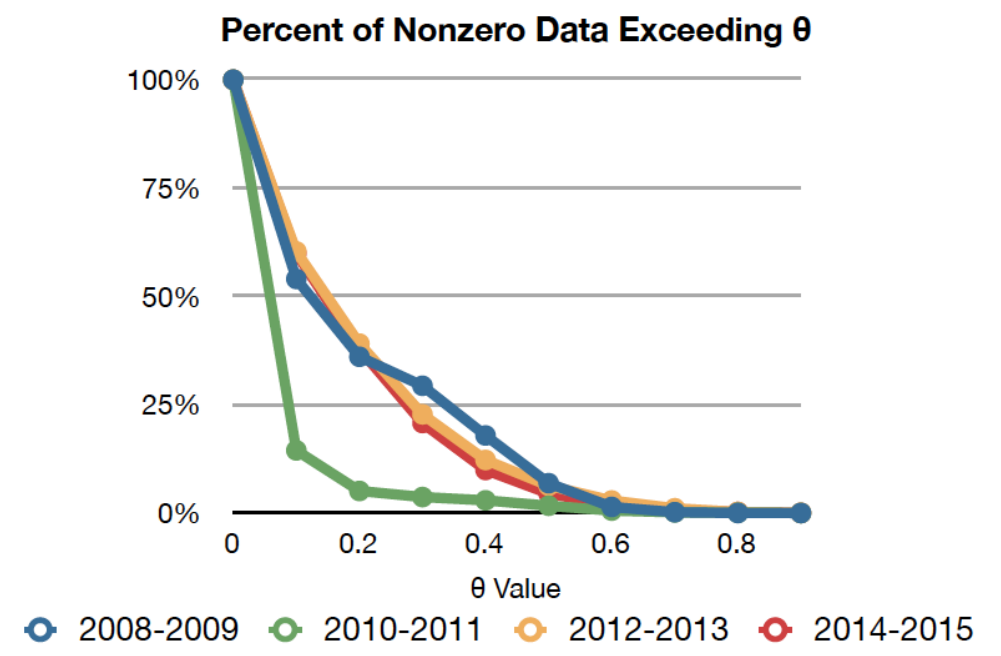

Figure 18: The percent of data remaining as $\theta$ increases is tracked.

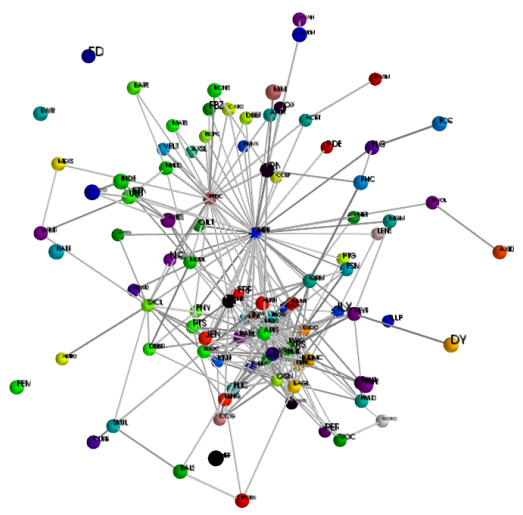

Figure 19: Example 3D graph we generated

\section{Appendix C: Tables}




\begin{tabular}{|l|l|l|l|l|l|l|l|l|l|}
\hline & $\theta=0.1$ & $\theta=0.2$ & $\theta=0.3$ & $\theta=0.4$ & $\theta=0.5$ & $\theta=0.6$ & $\theta=0.7$ & $\theta=0.8$ & $\theta=0.9$ \\
\hline $\mathbf{2 0 0 8 - 2 0 0 9}$ & $55.76 \%$ & $52.52 \%$ & $42.09 \%$ & $27.34 \%$ & $14.03 \%$ & $4.68 \%$ & $2.16 \%$ & $0.36 \%$ & $0.00 \%$ \\
\hline $\mathbf{2 0 1 0 - 2 0 1 1}$ & $18.41 \%$ & $16.97 \%$ & $15.88 \%$ & $12.76 \%$ & $7.58 \%$ & $3.01 \%$ & $1.08 \%$ & $0.72 \%$ & $0.24 \%$ \\
\hline $\mathbf{2 0 1 2 - 2 0 1 3}$ & $57.02 \%$ & $41.48 \%$ & $28.45 \%$ & $19.30 \%$ & $13.66 \%$ & $9.65 \%$ & $5.64 \%$ & $2.38 \%$ & $0.50 \%$ \\
\hline $\mathbf{2 0 1 4 - 2 0 1 5}$ & $57.04 \%$ & $39.52 \%$ & $24.10 \%$ & $15.12 \%$ & $9.88 \%$ & $6.44 \%$ & $4.34 \%$ & $2.69 \%$ & $0.75 \%$ \\
\hline
\end{tabular}

Figure 20: Degeneracy for $\rho>\theta$ as Percent of Max Degeneracy for Period

\begin{tabular}{|l|l|l|l|l|}
\hline & $\mathbf{2 0 0 8 - 0 9}$ & $\mathbf{2 0 1 0 - 1 1}$ & $\mathbf{2 0 1 2 - 1 3}$ & $\mathbf{2 0 1 4 - 1 5}$ \\
\hline $0.0 \leq \theta<0.1$ & 808656 & 754389 & 467342 & 584374 \\
\hline $0.1 \leq \theta<0.2$ & 13678 & 61476 & 134492 & 97544 \\
\hline $0.2 \leq \theta<0.3$ & 5100 & 9232 & 103314 & 75602 \\
\hline $0.3 \leq \theta<0.4$ & 8736 & 5106 & 66600 & 47978 \\
\hline $0.4 \leq \theta<0.5$ & 8384 & 8208 & 37570 & 24036 \\
\hline $0.5 \leq \theta<0.6$ & 4178 & 7310 & 21738 & 11756 \\
\hline $0.6 \leq \theta<0.7$ & 928 & 2672 & 11304 & 5144 \\
\hline $0.7 \leq \theta<0.8$ & 138 & 710 & 5408 & 1942 \\
\hline $0.8 \leq \theta<0.9$ & 8 & 346 & 1430 & 878 \\
\hline $0.9 \leq \theta<1.0$ & 0 & 52 & 126 & 166 \\
\hline
\end{tabular}

Figure 21: Edge Distribution

Symbol Name

AXAS Abraxas Petroleum Corporation

AE Adams Resources \& Energy, Inc.

AAV Advantage Oil \& Gas Ltd

ANW Aegean Marine Petroleum Network Inc.

AHGP Alliance Holdings GP, L.P.

ARLP Alliance Resource Partners, L.P.

ALJ Alon USA Energy, Inc.

ALDW Alon USA Partners, LP

AETI American Electric Technologies, Inc.

AEUA Anadarko Petroleum Corporation

APC Anadarko Petroleum Corporation

AR Antero Resources Corporation

APA Apache Corporation

AREX Approach Resources Inc.

$\begin{array}{llll}\text { LastSale } & \text { MarketCap } & \text { IPOyear } & \text { Sector } \\ 1.14 & 121234441.14 & \mathrm{n} / \mathrm{a} & \text { Energy } \\ 39.1 & 164908003.6 & \mathrm{n} / \mathrm{a} & \text { Energy } \\ 5.45 & 1004931696.65 & \mathrm{n} / \mathrm{a} & \text { Energy } \\ 7.62 & 367827709.86 & 2006 & \text { Energy } \\ 14.47 & 866217610 & 2006 & \text { Energy } \\ 12.88 & 957950322 & 1999 & \text { Energy } \\ 11.37 & 808143306.96 & 2005 & \text { Energy } \\ 14.13 & 883266851.07 & 2012 & \text { Energy } \\ 2.4295 & 20053095.4295 & \mathrm{n} / \mathrm{a} & \text { Energy } \\ 32.76 & 0 & 2015 & \text { Energy } \\ 48.76 & 24791468427.72 & \mathrm{n} / \mathrm{a} & \text { Energy } \\ 24.75 & 6857268066 & 2013 & \text { Energy } \\ 49.63 & 18774919019.92 & \mathrm{n} / \mathrm{a} & \text { Energy } \\ 1.56 & 63714749.28 & 2007 & \text { Energy }\end{array}$

Figure 22: Example table of stocks for each sector; these stocks are in the Energy sector. 